\title{
Sensor-based Navigation and Integrated Control of Ambient Intelligent Wheeled Robots with Tire-Ground Interaction Uncertainties
}

\author{
Aleksandar Rodic ${ }^{1}$, Gyula Mester ${ }^{2}$ \\ ${ }^{1}$ University of Belgrade, Institute Mihajlo Pupin, Robotics Laboratory \\ Volgina 15, 11060 Belgrade, Serbia, aleksandar.rodic@pupin.rs \\ ${ }^{2}$ University of Szeged, Faculty of Engineering, Robotics Laboratory \\ Mars tér 7, 6724 Szeged, Hungary, gmester@inf.u-szeged.hu
}

\begin{abstract}
This paper regards the synthesis of intelligent non-visual sensor-based navigation, motion planning and the integrated control of indoor ambient adaptive wheelbased mobile robots in unknown environments with tire-ground interaction uncertainties. The problem relates to searching appropriate techniques how to navigate towards a target position in an unknown environment when the obstacles to avoid are discovered in real time, and how to maintain collision free motion of a high dynamic performance. Environments characterized by variable ground surface conditions with immobile obstacles of different shapes and sizes will be considered in the paper as unexpected disturbances, i.e. system uncertainties. The tools developed to address this issue thus consist of the combination of cognitive motion planning and control theory techniques, including a nonlinear model-based approach. Two characteristic approaches to integrated control are evaluated in the paper: a kinematical as well as dynamic one, in the sense of control efficiency and robustness to the environmental and model uncertainties. Characteristic simulation tests are performed to verify the proposed algorithms.
\end{abstract}

Keywords: mobile robots; sensor-based navigation; integrated control; tire-ground interaction

\section{Introduction}

Mobile wheel-based robots are subjected to many recent research studies with aim to provide reliable and robust robotic platforms for broad service applications at home, in office, and at public institutions. Mobile robotic platforms form the basis for the building (development) of high-tech devices, such as personal robots of high performance to be widely used in the future in everyday human life. The problem of the use of such advanced intelligent systems is related to the success in solving complex cognitive and control tasks, such as intelligent navigation, 
motion planning and robust control of the system dynamics in conditions of unknown, unpredicted and evolving environments. The problem of ambient adaptation to the unstructured, confined and cluttered environments as well adaptation to the variable ground surface conditions (contingency risks) requires the development of efficient control techniques. A combined knowledge-based and model-based algorithm that couples navigation and control capabilities into a unified control architecture designed for the accurate system navigation and integrated control of wheeled robot dynamics is proposed in the paper. Autonomous mobile robots are required to have high dynamic performance, in the sense of dynamic, non-jerky and smooth motion, in order to ensure the reliable performance of tasks imposed.

Numerous research studies concerning control of wheeled mobile robots were reported in [1], [2]. In particular, non-holonomy constraints associated with these systems have motivated the development of highly nonlinear control techniques. For the sake of simplicity, the control methods are developed mainly for car-like mobile robots. The heuristic methods were the first techniques used to generate motion based sensors. The majority of these works were derived from classic planning methods [3]. The methods of physical analogies assimilate obstacle avoidance to a known physical problem. The representative of them is the potential field method [4]. There are methods that compute some high-level information as intermediate information, which is translated next in motion. The nearness diagram navigation [5,6] is a representative of this method. In paper [7] a multi-agent, self-organizing system of mobile robots is controlled by the implementation of the genetic algorithm. The solution proposed in paper [8] represents an original approach to the design of the 2-DOF Takagi-Sugeno PIfuzzy controller based on the stability analysis theorem. For implementation of the ethologically inspired robot behavior, a platform based on a fuzzy state-machine was suggested in [9]. In paper [10], the method of utilization of the low-resolution data for control purposes was applied. In this paper, control is based on fuzzy logic, with the deployment of stochastic digital low-resolution time arrays. The imprecision of the control method was eliminated by stochastic noise superimposed during data gathering, while the negative effects of noise are suppressed both by the fuzzy nature of the decision-making process and by the energy inertia of the controlled object.

The considerations to be conducted in the paper will demonstrate a methodology of the motion control of mobile robots that combines ad-hoc motion planning and obstacle avoidance together with model-based, integrated control of the robotic system. The paper is organized as follows: Section 1: introduction. In Section 2, the modeling of wheel-based robots is presented. In Section 3, sensor-based navigation and motion planning are illustrated. In Section 4, the motion control is presented. Simulation examples and verification of the proposed methodology are illustrated in Section 5. Conclusions are given in Section 6. 


\section{Modeling of Wheel-based Robots}

For the purpose of control system development and the simulation model of a nonholonomic wheeled robot with differential (skid) steering is considered in the paper as presented in Fig. 1. A 2WD indoor mobile robotic platform with differential steering and two auxiliary (passive, i.e. non-powered) wheels is assumed in the paper as a system representative. A non-linear model of such a wheeled mobile robot is considered, taking into account that the robot can move on a sloped surface, too. In the general case, surface inclination angle can appear in both longitudinal $\gamma_{x}$ as well as lateral $\gamma_{y}$ direction of motion (Fig. 2) with respect to the longitudinal $\mathrm{x}$-axis of the robot body. The direction of the motion, i.e. the angle of the forward (transport) speed vector $\overrightarrow{\mathbf{V}}$ (Fig. 1), depends on amplitudes of particular tire angular velocities, rigid-body parameters as well as tire-ground interaction parameters and ground surface condition. The referent coordinate system $O X Y Z$ to be considered in the paper is attached to the ground surface. The local mobile coordinate system oxyz is attached to the mass center (MC) of the wheeled mobile robot. Robot motion is consequence of differential steering, i.e. controlled changing of tires r.p.m. corresponding longitudinal $F_{x i}, i=1,2$ and lateral $F_{y i}, i=1,2$ tire forces (Fig. 2), which cause the robot to move in the desired direction and with the desired forward speed. The passive auxiliary tires have zero traction forces $F_{x i}=0, i=3,4$. In the general case, when the auxiliary wheels (i.e. their axles) are not collinear with the active (powered) wheels, they can slide on the surface against the friction forces. In this case, the lateral tire forces $F_{y i} \neq 0, i=3,4$ appear on the auxiliary wheels. In the general case, the forward speed $\overrightarrow{\mathbf{V}}$ is not collinear with the direction of the longitudinal wheeled mobile robot axis of symmetry. The angle between the velocity vector $\overrightarrow{\mathbf{V}}$ and the longitudinal $\mathrm{x}$-axis of symmetry is defined by the angle $\beta$ known as the slip angle of vehicle [11]. The particular mobile robot wheels perform corresponding rotational as well linear movements. Linear tire velocities are signed by $\vec{v}_{i}, i=1, \ldots, 4$ in Fig. 1 . In the general case, these velocities do not coincide with the corresponding direction of robot motion defined by the vector $\overrightarrow{\mathbf{V}}$. The consequence of this is the appearance of tire slipping defined by the corresponding angles $\varsigma_{i}, i=1, \ldots, 4$ as presented in Fig. 1. Some important geometry parameters of the wheeled robot (rover) are presented in Fig. 1. These are: $b$ is the track of rover, $l_{x}$ is the relative position of the active wheels with respect to the robot mass centre (MC) observed along the longitudinal $x$ coordinate direction, $l_{f}$ and $l_{r}$ are corresponding distances of the auxilary wheels (front and rear) from the robot MC. 


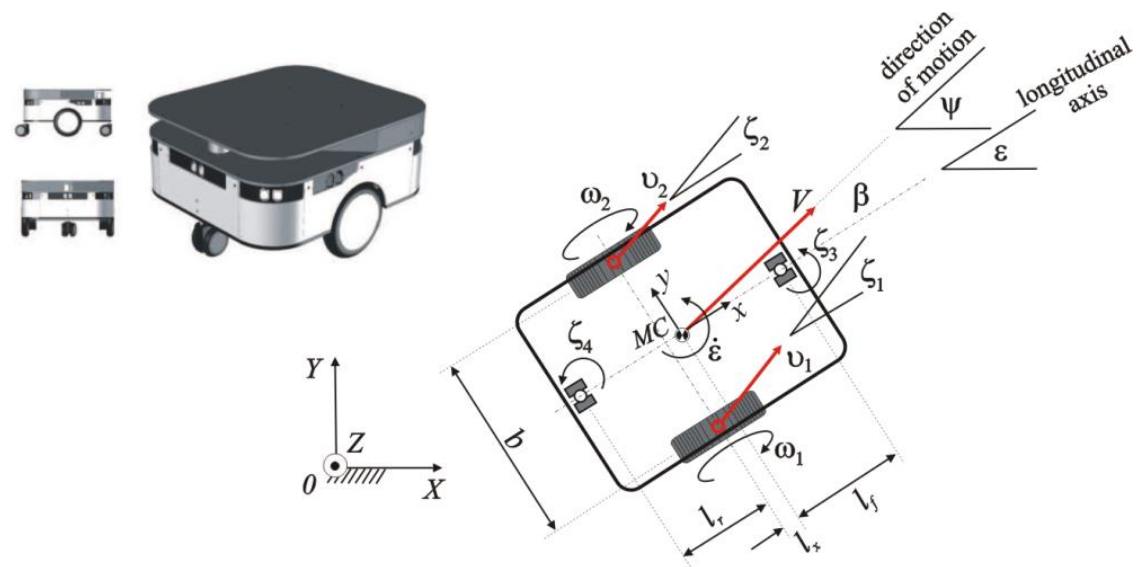

Figure 1

Industrial non-holonomic 2WD wheel-based mobile robot RobuLab10 [12]. Corresponding kinematic model of the assumed robotic platform and parameters of interest.

The vector of the state variables, expressed with respect to the referent coordinate system $O X Y Z$ can be written in the form:

$\mathbf{q}=\left[\begin{array}{lll}X & Y & \varepsilon\end{array}\right]^{T}$

where $X$ and $Y$ represent corresponding linear displacements (translations) of robot body $\mathrm{MC}$ determined in the absolute coordinate system attached to the ground surface, $\varepsilon$ is corresponding yaw angle, i.e. turning of the rover about the $\mathrm{Z}$-axis measured with respect to the $\mathrm{X}$-axis (Fig. 1).

\subsection{Kinematical Model of a 2WD Robot}

The kinematical model of the robot presented in Fig. 1, with two active and two auxiliary non-powered wheels can be defined by the following relation:

$$
\left[\begin{array}{c}
V \\
\dot{\varepsilon}
\end{array}\right]=\left[\begin{array}{cc}
r_{t} / 2 & r_{t} / 2 \\
r_{t} / b & -r_{t} / b
\end{array}\right] \cdot\left[\begin{array}{c}
\omega_{1} \\
\omega_{2}
\end{array}\right]
$$

where $V$ and $\dot{\varepsilon}$ represent corresponding amplitudes of the forward (transport) speed as well as yaw-rate of robot rigid-body, $r_{t}$ is tire radius, and $\omega_{1}$ and $\omega_{2}$ are corresponding right and left tire angular velocities. The transport speed $\overrightarrow{\mathbf{V}}$, determined in the plane of motion, has two components - the longitudinal $\dot{x}$ and lateral $\dot{y}$ :

$$
V=\|\overrightarrow{\mathbf{V}}\|=\sqrt{\dot{x}^{2}+\dot{y}^{2}}
$$


Two kinematical variables are needed for tire modeling: tire slip ratio $s_{i}$ and tire slip angle $\alpha_{i}$. These variables can be calculated $[11,12]$ for every particular robot tire using the following relations:

$$
\begin{aligned}
& s_{i}=\frac{v_{i} \cos \left(\alpha_{i}\right)-r_{i} \omega_{i}}{r_{i} \omega_{i}}, i=1, \ldots, 4 \\
& \alpha_{i}=-\zeta_{i}, i=1, \ldots, 4
\end{aligned}
$$

The powered tires $i=1,2$ have no possibility of steering in a direct way while the passive wheels $i=3,4$ are free for turning about the vertical axis to enable better system maneuverability (Fig. 1). The value $v_{i}$ is the corresponding linear speed of the centre of mass of the particular $i$-th robot tire, and $\zeta_{i}$ represents the so-called tire speed angle defined with respect to the longitudinal direction of motion collinear to the longitudinal $\mathrm{x}$-axis of symmetry. The translational (linear) tire speeds of tire mass centers are determined by the relations:

$v_{1}=\sqrt{\left(\dot{y}-l_{x} \dot{\varepsilon}\right)^{2}+(\dot{x}-b / 2 \dot{\varepsilon})^{2}}$ for right wheel

$v_{2}=\sqrt{\left(\dot{y}-l_{x} \dot{\varepsilon}\right)^{2}+(\dot{x}+b / 2 \dot{\varepsilon})^{2}}$ for left wheel

$v_{3}=\sqrt{\left(\dot{y}+l_{f} \dot{\varepsilon}\right)^{2}+\dot{x}^{2}} \quad$ for front wheel

$v_{4}=\sqrt{\left(\dot{y}-l_{r} \dot{\varepsilon}\right)^{2}+\dot{x}^{2}} \quad$ for rear wheel

where the directions of the particular tire velocities (6) are determined by the corresponding angles $\zeta_{i}$, which are calculated from the expressions:

$$
\begin{aligned}
& \operatorname{tg}\left(\zeta_{1}\right)=\frac{\dot{y}-l_{x} \dot{\varepsilon}}{\dot{x}-b / 2 \dot{\varepsilon}}, \quad \operatorname{tg}\left(\zeta_{2}\right)=\frac{\dot{y}-l_{x} \dot{\varepsilon}}{\dot{x}+b / 2 \dot{\varepsilon}} \\
& \operatorname{tg}\left(\zeta_{3}\right)=\frac{\dot{y}+l_{f} \dot{\varepsilon}}{\dot{x}}, \quad \operatorname{tg}\left(\zeta_{4}\right)=\frac{\dot{y}-l_{r} \dot{\varepsilon}}{\dot{x}}
\end{aligned}
$$

\subsection{Model of 2WD Robot Rigid-Body Dynamics}

The model of rigid-body dynamics of the assumed 2WD mobile robot (Fig. 1) is presented in Fig. 2. The dynamic model of this robotic system can be defined in the following form:

$$
\mathbf{T}=\mathbf{H}(\mathbf{q}) \cdot \ddot{\mathbf{q}}+\mathbf{h}_{\mathrm{ccg}}(\mathbf{q}, \dot{\mathbf{q}})-\mathbf{F}_{\mathrm{w}}(\dot{\mathbf{q}})-\mathbf{J}_{\mathrm{s}} \cdot \mathbf{S}
$$

where $\mathbf{T} \in \mathfrak{R}^{3 \times 1}$ is a vector of the generalized forces/torques acting in robot MC. The vector $\mathbf{T}$ has three components collinear to the main coordinate directions $X$, $Y$ and $Z$ (Fig. 2): the generalized forces $T_{X}$ and $T_{Y}$ and corresponding generalized 
torque $T_{\varepsilon}$ about z-axis; $\mathbf{H} \in \mathfrak{R}^{3 \times 3}$ is an inertia matrix of the robot-body; $\mathbf{h}_{\text {ccg }} \in \mathfrak{R}^{3 \times 1}$ is a vector of centrifugal, Coriolis and gravity forces; $\mathbf{F}_{\mathbf{w}} \in \mathfrak{R}^{3 \times 1}$ is a vector of external resistance forces and torques that includes aerodynamic resistance, rolling resistance and Coulomb friction forces. Body impact forces (moments) as the consequence of an occasional strike of the robot to the surrounding objects are also taken into account by the vector $\mathbf{S} \in \mathfrak{R}^{3 \times 1}$. Corresponding Jacobian is defined by $\mathbf{J}_{\mathbf{S}} \in \mathfrak{R}^{3 \times 3}$. The vector of generalized forces T, defined by (8), can be expressed with respect to the mobile coordinate system MC-xy. Then, it can be defined in the form:

$$
\begin{aligned}
& \boldsymbol{\tau}=\left[\begin{array}{c}
\tau_{x} \\
\tau_{y} \\
\tau_{\varepsilon}
\end{array}\right]=\left[\begin{array}{c}
\sum_{i=1}^{4} F_{x i} \\
\sum_{i=1}^{4} F_{y i} \\
M_{Z}
\end{array}\right] ; \quad F_{x 3}=0 ; \quad F_{x 4}=0 \\
& M_{Z}=\left(F_{x 1}-F_{x 2}\right) \cdot \frac{b}{2}+\left(F_{y 1}+F_{y 2}\right) \cdot l_{x}+F_{y 3} \cdot l_{f}-F_{y 4} \cdot l_{r}
\end{aligned}
$$

where $F_{x_{i}}$ and $F_{y_{i}}(\mathrm{i}=1, . ., 4)$ are the corresponding longitudinal and lateral traction (braking) tire forces (Fig. 2) while $b, l_{x}, l_{f}$ and $l_{r}$ are the constructive parameters presented in Fig. 1.
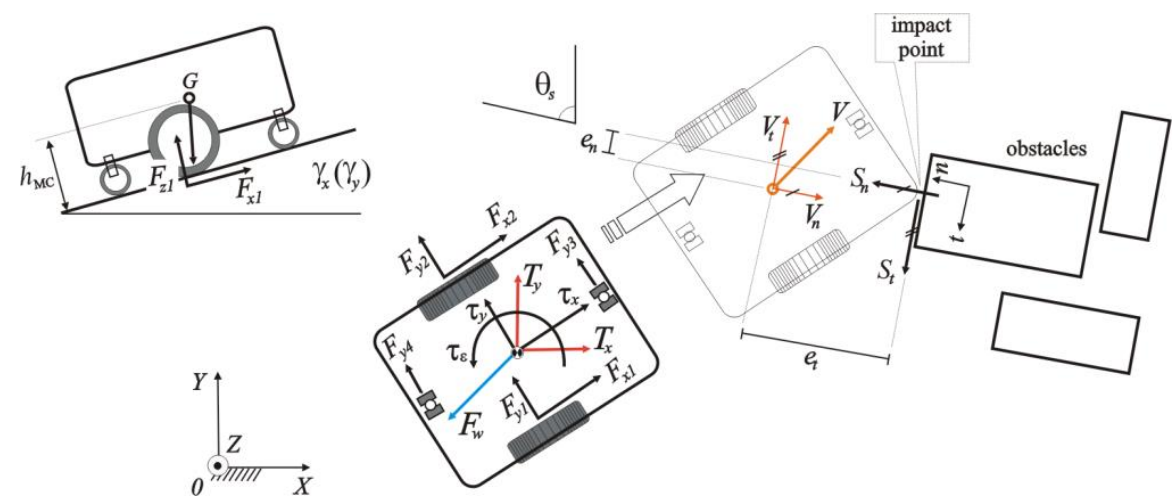

Figure 2

Dynamic model of the assumed 2WD wheel-based robot including traction (braking) forces, resistance forces, side impact forces and slope effects

The relation between the generalized forces and torques $\mathbf{T}$ expressed in the absolute coordinate system $0 X Y Z$ and corresponding forces and torques $\boldsymbol{\tau}$ defined in the local coordinate system MC-xyz can be defined in the following way: 
$T_{X}=\tau_{x} \cos (\varepsilon)-\tau_{y} \sin (\varepsilon)$,

$T_{Y}=\tau_{x} \sin (\varepsilon)+\tau_{y} \cos (\varepsilon)$,

$T_{\varepsilon}=\tau_{\varepsilon}$

The corresponding matrix and vectors given in (8) are assumed in the form [11]:

$$
\begin{aligned}
\mathbf{H} & =\left[\begin{array}{ccc}
m & 0 & 0 \\
0 & m & 0 \\
0 & 0 & I_{z}
\end{array}\right] \\
\mathbf{h}_{\text {ccg }} & =\left[\begin{array}{c}
-m \dot{y} \dot{\varepsilon}+m g \sin \left(\gamma_{x}\right) \\
m \dot{x} \dot{\varepsilon}+m g \sin \left(\gamma_{y}\right) \\
0
\end{array}\right]
\end{aligned}
$$

where $m$ is the lump mass of robot system, $I_{z}$ is the robot's axial moment of inertia with respect to the $\mathrm{z}$ - axis, and $\mathrm{g}$ is the magnitude of gravity acceleration. The resultant vector of the aerodynamic resistance as well as the rolling resistance forces and torques is calculated in a way [11]:

$$
\mathbf{F}_{\mathbf{w}}=\left[\begin{array}{c}
-K_{x} \dot{x}^{2}-\sum_{i=1}^{4} f_{r_{i}} F_{z_{i}} \cos \zeta_{i} \\
-K_{y} \dot{y}^{2}-\sum_{i=1}^{4} f_{r_{i}} F_{z_{i}} \sin \zeta_{i} \\
M_{\alpha}
\end{array}\right]
$$

where $\mathrm{K}_{\mathrm{x}}, \mathrm{K}_{\mathrm{y}}$ represents corresponding air resistance coefficients of robot body; $M_{\alpha}$ is a sum of tire self-aligning torques (slipping resistances about the vertical axis) due to robot turning about vertical axis; $f_{r_{i}}$ is a rolling resistance coefficient of the i-th tire, and $F_{z_{i}}$ represents corresponding tire payload.

The impact force vector $\mathbf{S}$ has in a general case three particular components $\mathbf{S}=\left[S_{n} S_{t} S_{\varepsilon}\right]^{T}$ (Fig. 2). The variation of motion quantity exchanged during the robot strike in the particular impact point (Fig. 2) is equal to the impulse of the impact force $S_{n}$ produced in the direction $\vec{n}$. The tangential impact force component $S_{t}$ depends on strike magnitude and corresponding body friction coefficient. Bearing in mind what has been previously said, the following relations can be derived:

$$
\mathbf{S}_{\mathbf{n}}=\frac{m \cdot V \cdot \cos \left(\theta_{s}\right)}{\Delta t} \cdot \overrightarrow{\mathbf{n}}
$$


where $\Delta t$ represents the time interval of impact impulse. The tangential component of impact force $S_{t}$ is calculated from the relation:

$$
\mathbf{S}_{\mathbf{t}}=-\mu_{s} \cdot S_{n} \cdot \overrightarrow{\mathbf{t}}
$$

where $\mu_{s}$ is Coulomb's friction coefficient characteristic for a relative two bodies sliding, i.e. robot-object interaction. In the general case, an impact force causes additional rotation of the robot body due to the particular location $e_{n}$ of the impact point with respect to the MC (Fig. 2). Then, the turning moment of the impact force about the axis that passes through the MC can be defined in a way, taking into account that $\overrightarrow{\mathbf{p}}=\overrightarrow{\mathbf{n}} \times \overrightarrow{\mathbf{t}}$ :

$$
\mathbf{S}_{\varepsilon}=\left(S_{n} \cdot e_{n}-S_{t} \cdot e_{t}\right) \cdot \overrightarrow{\mathbf{p}}
$$

Jacobian $\mathbf{J}_{\mathbf{s}}$ in (8) is determined in a form of the transformation matrix that is calculated for the case of system rotation about the z-axis for the angle $\theta_{s}$ as the relative angle between local $x-y$ and $n-t$ coordinate systems (see Fig. 2). In this case, the impact forces and torques $\mathbf{J}_{\mathbf{s}} \cdot \mathbf{S}$ in (8) can be calculated by the relation:

$$
\mathbf{J}_{\mathbf{s}} \cdot \mathbf{S}=\left[\begin{array}{ccc}
-\sin \left(\theta_{s}\right) & \cos \left(\theta_{s}\right) & 0 \\
\sin \left(\theta_{s}\right) & \cos \left(\theta_{s}\right) & 0 \\
0 & 0 & 1
\end{array}\right] \cdot\left[\begin{array}{l}
S_{n} \\
S_{t} \\
S_{\varepsilon}
\end{array}\right]
$$

\subsection{Non-linear Tire Model}

A considerable number of different models of tire force and moment generating properties have been proposed in the available literature. The standard description of vehicle tire dynamics is the so called the magic formula tire model originally introduced by Pacejka and Bakker [13]. The model provides a set of mathematical formulae from which the forces and moment acting from road to tire can be calculated at longitudinal and lateral slip conditions, which may occur simultaneously. The formula (model) expresses the side force $F_{y}$, the aligning torque $M_{\alpha}$ and the longitudinal force $F_{x}$ as a function of two arguments - the side slip angle defined by (5) and the longitudinal tire slip ratio determined by (4), respectively. The general form of the formula, which holds for a given value of vertical tire load, looks like:

$$
f(u)=D \cdot \sin \{C \cdot \operatorname{arctg}[B \cdot u-E \cdot(B \cdot u-\operatorname{arctg}(B \cdot u))]\}
$$

The empiric non-linear Pacejka's tire model is shown in Fig. 3. For constant coefficients B, C, D and E the curve exhibits an anti-symmetric shape with respect to the origin. The formula is capable of producing characteristics which closely 
match measured curves for longitudinal $F_{x}$, side (lateral) $F_{y}$ force and selfaligning torque $M_{\alpha}$ as functions of their respective slip quantities: the slip angle $\alpha$ and longitudinal slip ratio $s$. The output variable stands for either $F_{x}, F_{y}$ or $M_{\alpha}$ and the input $u$ may represents $s$ or $\alpha$.

Tire payload, i.e. tire torques of rotation, can be now calculated from the relation:

$$
\tau_{t}=F_{x} \cdot r_{t}
$$

taking into account the longitudinal tire force $F_{x}$ and tire radius $r_{t}$. Tire payload $\tau_{t}$ as well as tire angular velocity are used as feedback signals for servo-control of wheeled robot motion.

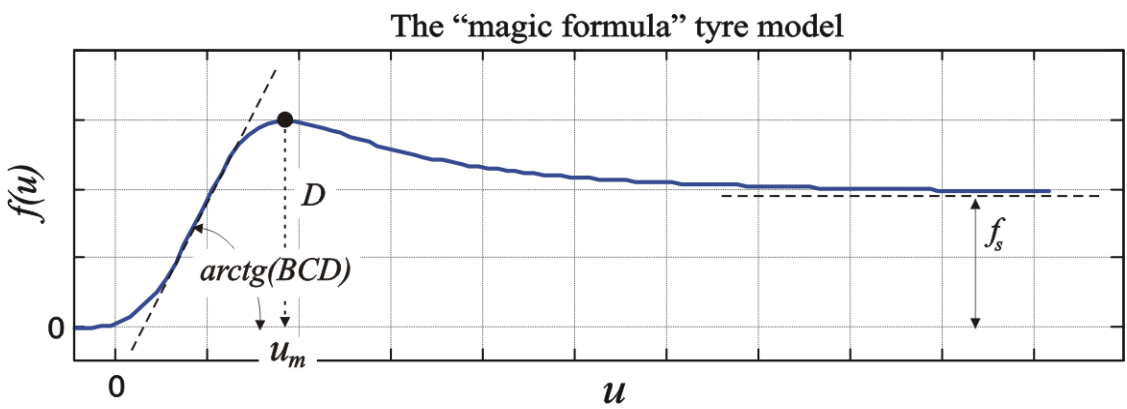

Figure 3

The Magic formula tire model - a graphic presentation

The model of wheeled robot determined by relations $(2-20)$ is used in the chapter for synthesis of algorithms for control of robot dynamics in the case of variation of tire-ground adhesion parameters, i.e. in the presence of tire-ground interaction uncertainties.

\section{Sensor-based Navigation and Motion Planning}

The objective of motion planning techniques is to compute a collision-free trajectory to the target configuration that complies with the vehicle constraints (Fig. 4). The objective is to move a vehicle towards a target location free of collisions with the obstacles detected by the sensors during motion execution. The advantage of reactive obstacle avoidance is to compute motion by introducing the sensor information within the control loop, used to adapt the motion to any contingency incompatible with the initial plans.

The approach that is elaborated in this section assumes that the robot is equipped with the corresponding sensors for the detection of obstacles (sonar proximity 
sensors and range-finder sensors) in its surrounding, as well as that it operates in informatically structured environments [14]. This assumes it should be connected to a wireless sensor network that ensures accurate localization of robot and target point (Fig. 4) within the work-space at every time instant. When autonomous robot moves towards the target position and its sensors detect obstacle(s), a corresponding avoiding strategy should be activated. In that sense, robot motion can be described as a compromise between avoiding obstacles and moving towards the target position as presented in Fig. 4. Autonomous robots react to both of the sensed variables (target direction and collision free direction of motion with respect to neighbourhood obstacles) to perform autonomous motion.

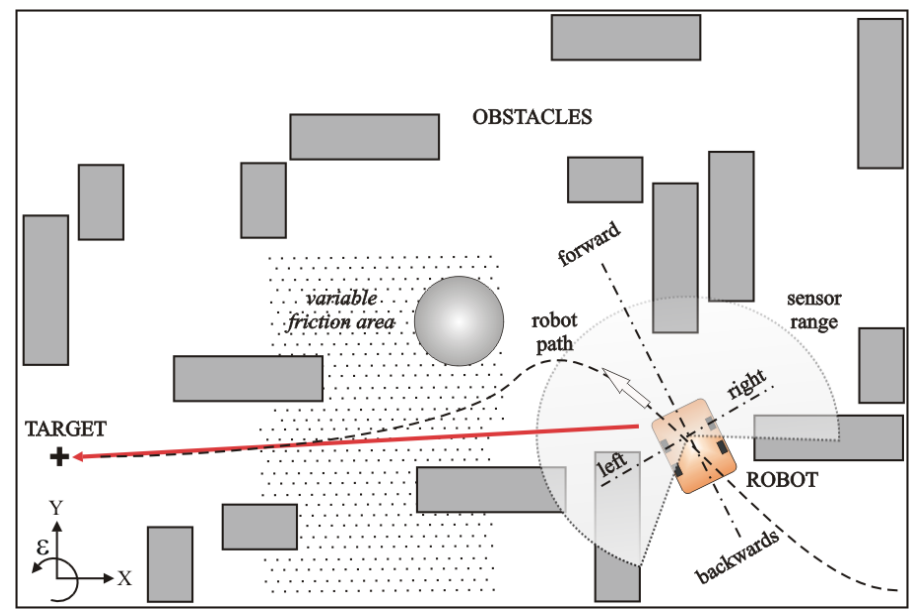

Figure 4

Model of sensor-based navigation and motion planning in presence of obstacles with variable tireground interaction conditions

For accurate navigation and motion planning in the presence of obstacles, the robot needs to sense the following variables (see Fig. 4): (i) distance to surrounding obstacle(s) within the sensor range (front-rear and right-left sectors of acquiring information), (ii) corresponding relative position of obstacles (i.e. angles measured relatively to the robot longitudinal $\mathrm{x}$-axis of symmetry), and (iii) relative position (azimuth angle) of robot with respect to the target point. Moving towards the target point and avoiding obstacles along the path predicted (Fig. 4), a mobile robot changes its orientation in the work-space as well as its forward velocity. When an obstacle is detected by sensors, the mobile robot slows down and changes its direction of motion according to the actual conditions detected. The navigation strategy of mobile robot is set to enable the automatic guidance of robot in the presence of obstacles and the accurate tracking of the estimated target direction. For the purpose of spatial reasoning in unknown and confined environments, an appropriate fuzzy inference system (FIS) is commonly used technique [14-17] to support such kind of cognitive tasks. In this paper, the FIS 
system presented in Fig. 5 is designed for collision free robot guidance in unknown environments with the presence of obstacles of different shapes and sizes.

The fuzzy navigation system, assumed in the paper to navigate robot in unknown, informatically structured environments, is shown in Fig. 5. It represents a multiinput / multi-output (MIMO) system with six inputs and two output ports.

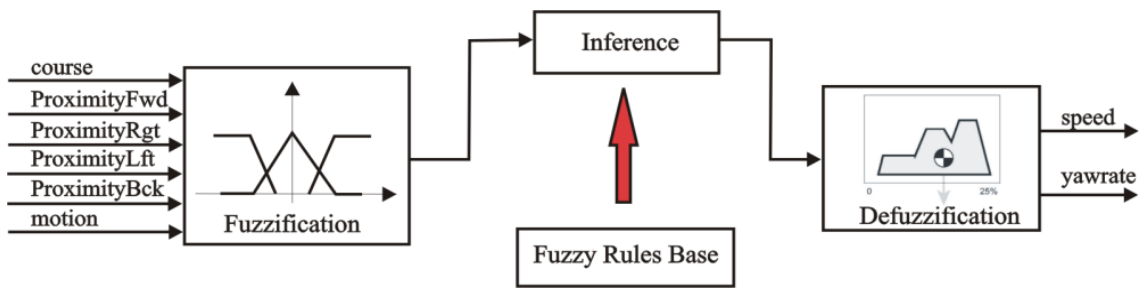

Figure 5

Block scheme of the Fuzzy Inference System developed for sensor-based navigation and obstacle avoidance in unknown environments

The following input variables are needed to be acquired from the work-space: target direction (azimuth angle of the referent course towards to the goal position); the "course" angle is determined relatively to the local coordinate system attached to the robot $\mathrm{MC}$, with $\mathrm{x}$-axis oriented along the longitudinal axis of robot platform (Fig. 1). The course is positive when the robot has to turn in left and negative when it moves right; proximity (distance) to obstacles in the forward direction; side proximity to obstacles (right-hand side and left-hand side direction); proximity to obstacles with respect to backwards direction; and indicator of motion concerning movement forward, backwards or standby status.

The corresponding output variables of the FIS considered in Fig. 5 are forward speed and yaw-rate of the wheeled robot. The FIS membership functions (MF) that correspond to the particular fuzzy input ports are presented in Figs. 6a-6d. The corresponding MF that corresponds to the output fuzzy ports are shown in Figs. 6e and 6f. The MFs chosen in this robot task have predominantly Gauss form.

The FIS rule database consists of 13 rules that allow the system to navigate properly in the presence of immobile obstacles. The rule data-base is systematized in Tab. 1. Weighting factors related to the particular rules are set to the unit value (Tab. 1). Membership functions and fuzzy rules are designed in the Fuzzy Logic Toolbox of Matlab/Simulink. 


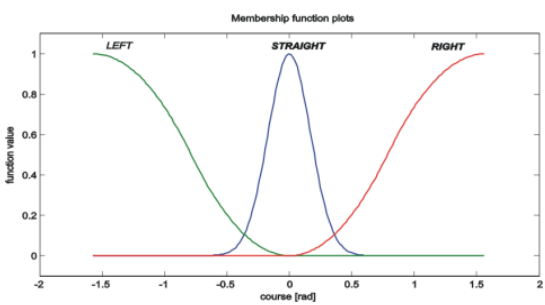

a)

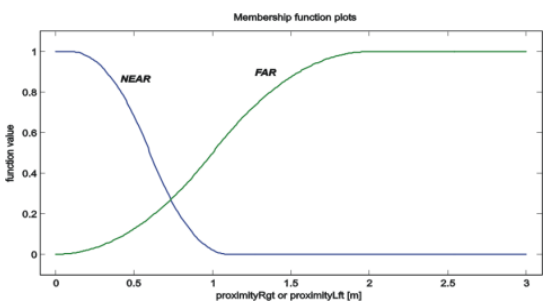

c)

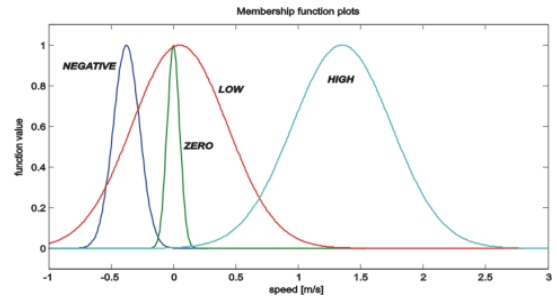

e)

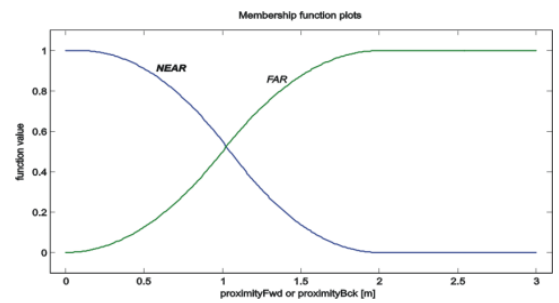

b)

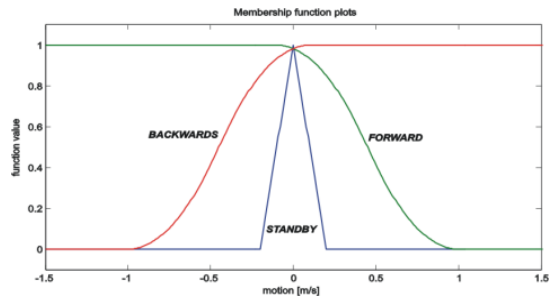

d)

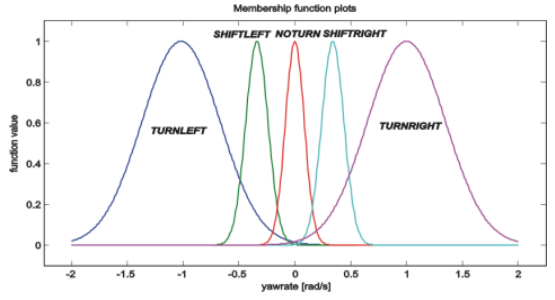

f)

Figure 6

Fuzzy membership functions used for sensor-based navigation input variable: a) "course" [-1.57, $1.57 \mathrm{rad}]$, b) distance from the obstacles in forward/backwards direction, "proximityFwd" or "proximityBck", [0,3 m], c) distance to obstacles in side directions right and left,"proximityRgt or proximityLft", [0, $3 \mathrm{~m}]$, d) status of motion, "motion", [-1.5, $1.5 \mathrm{~m} / \mathrm{s}]$, output variable: e) forward speed "speed", [-1, $3 \mathrm{~m} / \mathrm{s}], \mathrm{f})$ yaw-rate "yawrate" [-2, $2 \mathrm{rad} / \mathrm{s}]$.

Table 1

Data-base fuzzy rules designed to ensure robot navigation in unknown environments with obstacles of different geometry and size

\begin{tabular}{|c|c|c|c|c|c|c|c|c|c|}
\hline \multirow[t]{2}{*}{ Rule no. } & \multicolumn{6}{|c|}{ Fuzzy input variables } & \multicolumn{2}{|c|}{ Fuzzy output variables } & \multirow[t]{2}{*}{ Weight } \\
\hline & course & proximityFwd & proximity Bck & proximity $R g t$ & proximityLft & motion & speed & yawrate & \\
\hline 1 & STRAIGHT & FAR & & & & FORWARD & $\mathrm{HIGH}$ & NOTURN & 1 \\
\hline 2 & STRAIGHT & NEAR & & & & FORWARD & Low & NOTURN & 1 \\
\hline 3 & RIGHT & & & NO NEAR & & FORWARD & LOW & TURNRIGHT & 1 \\
\hline 4 & LEFT & & & & NO NEAR & FORWARD & LOW & TURNLEFT & 1 \\
\hline 5 & & & & NEAR & NEAR & FORWARD & Low & NOTURN & 1 \\
\hline 6 & & & & NEAR & NO NEAR & FORWARD & NO HIGH & SHIFTLEFT & 1 \\
\hline 7 & & & & NO NEAR & NEAR & FORWARD & NO HIGH & SHIFTRIGHT & 1 \\
\hline 8 & & & NO NEAR & & & NO FORWARD & NEGATIVE & & 1 \\
\hline 9 & & NEAR & NEAR & NO NEAR & NO NEAR & & LOW & SHIFTRIGHT & 1 \\
\hline 10 & & NEAR & NEAR & NEAR & NEAR & & ZERO & SHIFTRIGHT & 1 \\
\hline 11 & & NO NEAR & NEAR & NO NEAR & NO NEAR & FORWARD & $\mathrm{HIGH}$ & NOTURN & 1 \\
\hline 12 & RIGHT & NEAR & & NO NEAR & & FORWARD & LOW & TURNRIGHT & 1 \\
\hline \multirow[t]{2}{*}{13} & LEFT & NEAR & & & NO NEAR & FORWARD & LOW & TURNLEFT & 1 \\
\hline & \multicolumn{6}{|c|}{ connection type "AND" } & \multicolumn{2}{|c|}{ connection type "AND" } & \\
\hline
\end{tabular}




\section{Motion Control}

The control architecture of the wheeled mobile robot considered in the paper represents a modular hierarchy distributed structure. The proposed control system has two hierarchy levels, high and low. The high control level consists of a cognitive block (a knowledge-based block based on the fuzzy inference system presented in Fig. 5) coupled with a complementary model-based module. Such a controller takes into account the dynamics of the entire robotic system (2)-(20), including robot rigid-body dynamics, tire non-linear dynamics and tire-ground interaction effects (slipping, rolling resistance, etc.). The high control block is charged for sensor data acquisition, signal processing, sensor data fusion, sensorbased navigation, motion planning and the control of robot dynamics as well the appropriate control load distribution per particular robot wheels.

The low control block ensures the servo-control of DC-motors, whose task is the regulation of tire load (traction or braking torques) and tire angular velocities. In order to build the control for the wheeled mobile robot (Fig. 1) considered in the paper, the following assumptions have to be satisfied: (i) The model presented by relations (2 - 20) describes the system's physical behavior with satisfactory accuracy. (ii) The parameters of the model are acquired directly from the system by measurement or by estimation using corresponding sensor-based acquired information. (iii) In every sampling time it is possible to determine in a precise way the location (position) of the robot in the work-space as well as the location of the target point (Fig. 4) using corresponding localization sensors. (iv) Corresponding tire-ground interaction parameters (slipping and rolling resistance coefficients) can be estimated by use of the model described in Section 2. (v) Proximity sensors detect obstacles in the range of two meters with satisfactory accuracy.

If the previously listed assumptions are satisfied, the control system proposed in the paper is capable of ensuring high dynamic performance as well precise tracking of the target direction (Fig. 4). Concerning the accuracy of the model presented in Section 2, the relations $(2$ - 20) describe the entire system behavior (physics) including its empiric tire non-liner model. The tires of wheeled robots are factors that cause potential uncertainties in the system. Due to these reasons, their influence upon the system behavior is significant, and it is very important for the control efficiency that the tires' actual dynamics be modeled in an appropriate way. The parameters of rigid body model can be identified directly via measurement of the system or calculated based on appropriate measurements (e.g. robot mass, moments of inertia, dimensions, etc.). Tire-ground interaction parameters (adhesion parameters) are also estimated using appropriate experimental measurements and relations describing tire non-linear model.

The control algorithm of the wheeled mobile robot has to provide accurate path tracking and high dynamic performances of entire system. A control algorithm capable of providing such performance can be written in the following form [11]: 


$$
\begin{aligned}
& \mathbf{T}=\mathbf{H}(\mathbf{q}) \cdot \hat{\hat{\mathbf{q}}}+\mathbf{h}_{\mathrm{ccg}}(\mathbf{q}, \dot{\mathbf{q}})-\mathbf{F}_{\mathrm{w}}(\dot{\mathbf{q}}), \\
& \hat{\mathbf{q}}=\ddot{\mathbf{q}}_{\mathbf{0}}-\mathbf{K}_{\mathrm{d}} \cdot\left(\dot{\mathbf{q}}-\dot{\mathbf{q}}_{\mathbf{0}}\right)-\mathbf{K}_{\mathbf{p}}\left(\mathbf{q}-\mathbf{q}_{\mathbf{0}}\right)
\end{aligned}
$$

where $\mathbf{K}_{\mathbf{p}}$ and $\mathbf{K}_{\mathbf{d}}$ are corresponding matrices of the proportional and differential control gains, while the other values appearing in (21) have been already explained in (8). From (11) the generalized forces $T_{X}, T_{Y}$ and turning (spinning) torque $T_{\varepsilon}$ are determined with respect to the absolute coordinate system (Fig. 1). Then, corresponding driving forces and torques in the longitudinal $\tau_{x}$, lateral $\tau_{y}$ and yaw $\tau_{\varepsilon}$ direction are calculated from the relations:

$$
\begin{aligned}
& \tau_{x}=T_{X} \cos (\varepsilon)+T_{Y} \sin (\varepsilon), \\
& \tau_{y}=-T_{X} \sin (\varepsilon)+T_{Y} \cos (\varepsilon), \\
& \tau_{\varepsilon}=T_{\varepsilon}
\end{aligned}
$$

Generalized forces $\tau_{x}, \tau_{y}$ and torque $\tau_{\varepsilon}$ are produced by corresponding tire forces $F_{x}$ and $F_{y}$ acting in the longitudinal and side directions (Fig. 2). The 2WD rover must be considered as an "over controlled" system since in the considered particular case there are four tire forces $\left(F_{x 1}, F_{y 1}, F_{x 2}\right.$ and $F_{y 2}$, shown in Fig. 2) while the global robot motion is performed in three particular coordinate directions: $\mathrm{x}, \mathrm{y}$ and $\varepsilon$. The unknown forces $F_{x i}, i=1,2$ and $F_{y i}, i=1,2$ can be calculated indirectly (in a reverse way) from the relations (9) and (10), taking into account pre-determined generalized forces/torque $\tau_{x}, \tau_{y}$ and $\tau_{\varepsilon}$ from (22). In order to perform one such procedure some additional relation must be provided to enable the calculation (by elimination of one unknown variable) of unknown tire forces $F_{x 1}, F_{y 1}, F_{x 2}$ and $F_{y 2}$. This auxiliary relation that enables a decrease in number of unknown variables is:

$$
F_{y 2}=\kappa_{2} \cdot F_{y 1}
$$

where $\kappa_{2}=F_{y 2} / F_{y 1}$ is the corresponding ratio (coefficient) that defines the relationship between the particular side force amplitudes $F_{y 1}$ and $F_{y 2}$ of the right and left side wheels. Taking into account relation (23), as well including it into (9) and (10), the corresponding three equations upon the three unknown variables $F_{x 1}, F_{x 2}$ and $F_{y 1}$ can be solved from:

$$
\begin{aligned}
& \tau_{x}=F_{x 1}+F_{x 2}, \\
& \tau_{y}=\left(1+\kappa_{2}\right) \cdot F_{y 1}+F_{y 3}+F_{y 4}, \\
& \tau_{\varepsilon}=b \cdot F_{x 1}-b \cdot F_{x 2}+\left(1+\kappa_{2}\right) \cdot l_{x} \cdot F_{y 1}+F_{y 3} \cdot l_{f}-F_{y 4} \cdot l_{r}
\end{aligned}
$$


From (24) and auxiliary term (23) the unknown tire forces $F_{x i}, i=1,2$ and $F_{y i}, i=1,2$ are calculated. The tire forces $F_{y i}, i=3,4$ appearing on the auxiliary tires are considered as disturbance of the system. The actual control variables of the wheeled mobile robot are not tire forces but particular angular velocities of the active wheels $\omega_{i}, i=1,2$ (Fig. 1). Since the longitudinal $F_{x i}, i=1,2$ and lateral tire forces $F_{y i}, i=1,2$ are non-linear functions of the arguments, such as tire slip ratio $s_{i}$ and tire slip angle $\alpha_{i}$, this implies the necessity of an inverse procedure to be conducted in order to determine the actual control variables $\omega_{i}, i=1,2$. Accordingly, the corresponding tire slip ratios $s_{i}, i=1,2$ and tire slip angles $\alpha_{i}, i=1,2$ should be calculated before.

Theoretical aspects of modeling, spatial navigation, motion planning and control of robot dynamics considered in Sections 2, 3 and 4 will be verified in Section 5.

\section{Verification of the Proposed Methodology}

In order to verify the proposed navigation and control methodology, several characteristic simulation experiments are performed and analyzed in this section. An industrial, middle size indoor mobile robot platform RobuLab-10 (Fig. 1) is assumed for the purpose of simulation experiments whose model parameters are taken from the corresponding product-sheet [12] and given in the Appendix of the paper. The robot chosen is simulated moving in conditions of unknown environment, modeled as kind of an indoor labyrinth scenario (Fig. 7), and in conditions of confined work-space with the appearance of different ambient contingency risks, such as: variable tire-ground interaction characteristics (e.g. variable slipping coefficient and rolling resistance) and irregular geometry (e.g. obstacle shapes the and sizes of surrounding obstacles). For this purpose, the Virtual WRSN, a specialized modeling/simulation software toolbox for Matlab/Simulink, was used [14].

The first considered simulation example brings together and compares the characteristics of two concurrent control approaches to be applied with mobile wheel-based robots, kinematic (differential steering) and a dynamic (skid steering) approach. Differential steering is based on the calculation of referent right and left tire angular velocities and directly from (2), neglecting the effects of slipping of the robot tires and neglecting their distinct non-linear nature. The concurrent skid steering approach takes into account the entire rigid-body robot dynamics, nonlinear tire dynamics and variable tire-ground interaction characteristics such as tire slipping and rolling resistance. 
In order to provide an appropriate comparison of two considered concurrent control approaches, the simulation conditions assumed should be equal (same) in both test cases. This means the same navigation strategy as well as the same motion conditions (tire adhesion characteristics) are considered during the simulation tests. The slipping coefficient in the considered simulation examples is assumed to be $\mu=0.75$. This corresponds to a dry, relatively high-adhesive tireground surface.

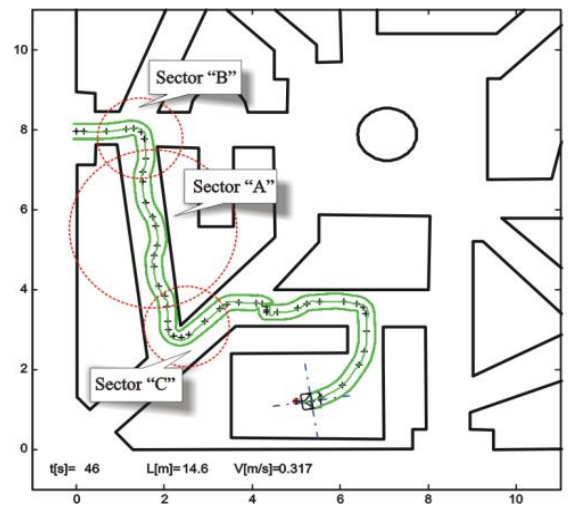

a)

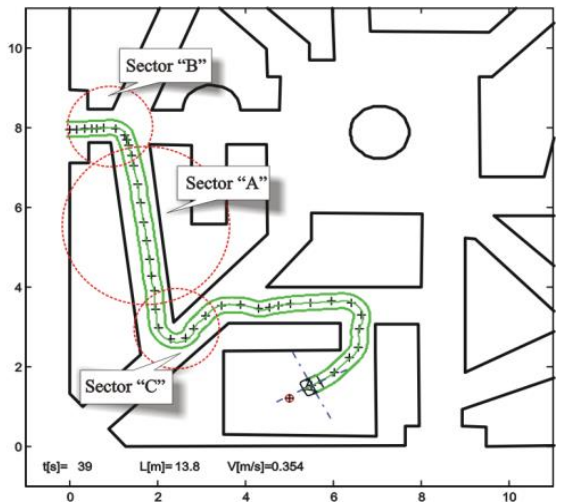

b)

Figure 7

Simulation of wheel traces of the mobile RobuLab-10 robot (Fig. 1) obtained in the cases:

a) path realized by control algorithm based on use of the kinematical approach, and b) path obtained by means of implementation of integrated (dynamic) control

The curvilinear motion of the mobile robot RobuLab-10 in an assumed labyrinth scenario (Fig. 7) was performed from the start-point towards the target-point along the target direction in the presence of surrounding obstacles of different shape and size. Under the same conditions, the robot-rover is controlled by use of dynamic control approach, too. The corresponding wheel traces of one such motion is shown in Figs. 7a and 7b. By comparison of performances of the two chosen test motions shown in Figs. $7 \mathrm{a}$ and $7 \mathrm{~b}$, better (smoother) motion appears in the case when a dynamic control is applied than in the case when a simplified kinematical algorithm is used. Consequently, robot with kinematicaly-based control makes the trip in approximately 46 (s) while the same robot needs less time (approximately 39 (s)) with the use of the dynamic control algorithm to perform the same task. The consequence is that the dynamically controlled robot moves noticeable faster than the same robot controlled in a kinematically-based way. The robot behaviour corresponding to the cases presented in Figs. $7 \mathrm{a}$ and $7 \mathrm{~b}$, is shown in Figs. 8a and $8 \mathrm{~b}$ by presentation of the corresponding state variables as characteristic system state indices.

With the analysis of these two motions, the following conclusion can be brought out. The dynamic control of robot motion ensures comparatively better system 
performance and maneuverability than the concurrent kinematically-based algorithm. It is characterized by more smooth and faster motion along the path. In addition, in cases of cornering manoeuvres (shown in Sectors B and C, Figs. 7a and $7 \mathrm{~b}$ ), the robot keeps (tracks) an imagined middle line of the corridor in a better way when it is controlled by the dynamic-based algorithm proposed in the paper.
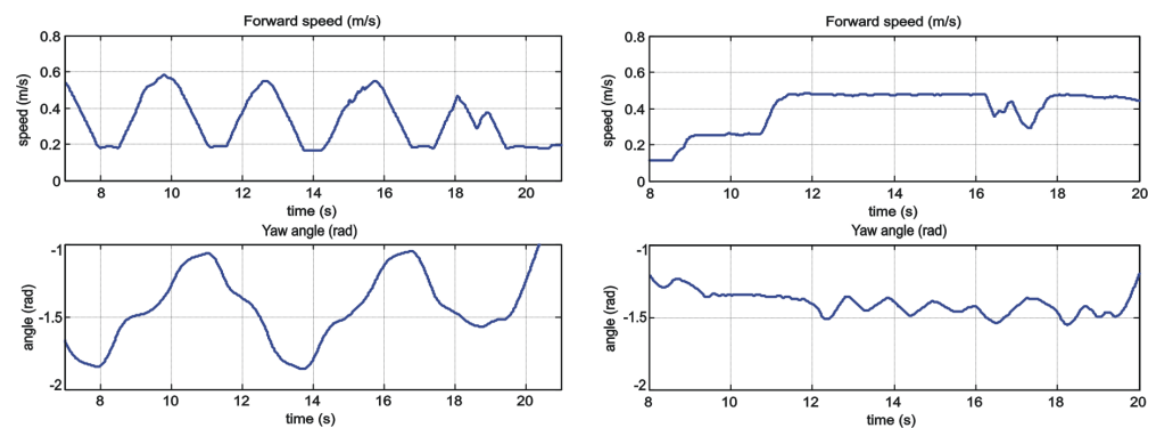

a)

b)

Figure 8

Robot state variables captured during the motion through the Sector "A" (Figs. 7): a) actual forward speed and yaw-rate of robot obtained in the case of kinematical approach applied to control robot motion, b) the same state indices obtained for the case of dynamic approach to control the same system

In such a way, by use of dynamic approach to control robot motion, less risk of collision with surrounding obstacles is attained, especially in the cornering manoeuvres. The reason why the dynamic control approach provides better system performance than the concurrent kinematical approach can be explained in the following way. By calculation of the referent tire angular velocities and directly from the robot kinematic model (2), the tire non-liner effects as well as the influence of tire-ground interaction factors are not taken in account.

The second simulation example considered in the paper regards the case when tire adhesion parameters vary during robot motion. Instead of the previously considered $\mu=0.75$, a new decreased friction coefficient of $\mu=0.45$ is introduced as a potential contingency risk of the robot motion. It corresponds to the case of a very slippery, low-friction floor surface. The corresponding simulation results that demonstrate one such dynamic behaviour are presented in Figs. 9a and 9b. Complementary simulation results presented in Figs. 9a and 9b are given to support the aforementioned theoretical considerations as well as to highlight the benefits of implementation of the proposed dynamic control with respect to the simplified kinematical approach to control motion.

Tire angular velocities are calculated by taking into account the linear tire speed, tire slip ratio and tire slip angle. The obtained right and left tire angular velocities $\omega_{i}, i=1,2$ for robot motion presented in Fig. 9 are shown in Fig. 10. 


\section{Conclusions}

The paper regards sensor-based, non-visual navigation and integrated control of indoor ambient adaptive wheel-based robots in unknown environments with contingency risks. The proposed architecture couples at the high functional level two specialized modules: a knowledge-based block and a model-based block. The cognitive knowledge-based block is synthesized for sensor-based navigation and spatial reasoning while the complementary model-based module is dedicated to the integrated control of robot motion and system dynamics.

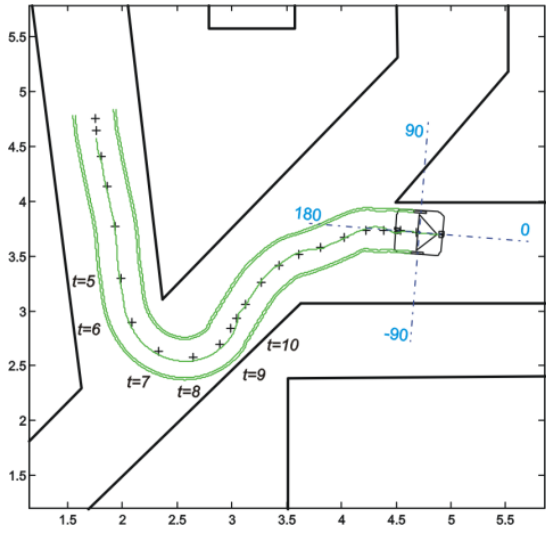

a)

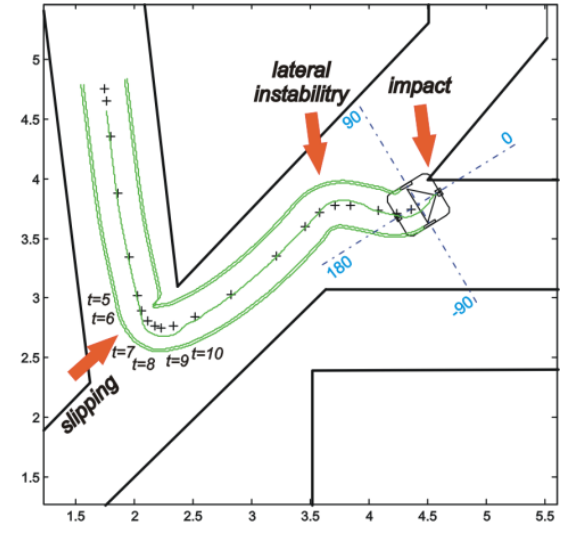

b)

Figure 9

Wheel traces obtained in simulation tests for a characteristic " $\mathrm{S}$ "cornering maneuver (Sector C, Figs.

$7 \mathrm{a}$ and $7 \mathrm{~b}$ ) in a case of low friction conditions (0.45): a) case when the system is controlled by implementation of dynamic, and b) simplified kinematical approach

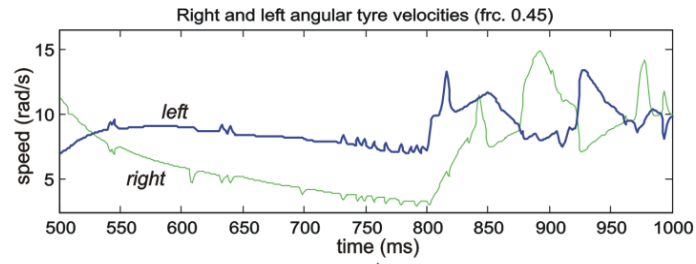

a)

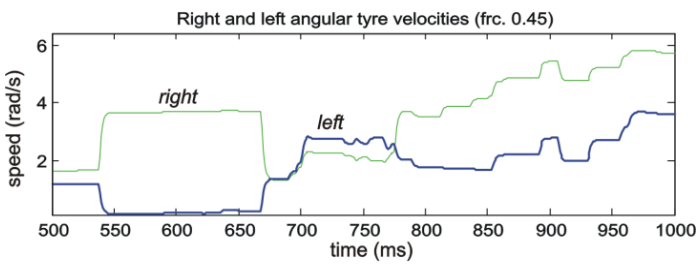

b)

Figure 10

Comparison of the tire angular velocities $\omega_{i}, i=1,2$ observed for a fragment of robot motion (Fig. 9) on the low-friction ground surface $(\mu=0.45)$ : a) dynamic, and b) kinematical control approach 
Navigation and control modules are coupled within a unique robot controller that is designed to ensure non-visual, target-oriented accurate navigation in unknown environments with contingency risks. The risks regard to variable tire-road interaction conditions, such as uncertainty in slipping conditions and rolling resistance as well as in variety of obstacle shapes and sizes. The cognitive navigation module must enable reliable, collision free motion in the presence of different obstacles. The proposed integrated control of a mobile robot is designed to improve dynamic performances (longitudinal and lateral stability, maneuverability in a confined and cluttered environment, etc.) of the robotic system and to ensure the accurate tracking of target direction towards a goal point. The paper considers the non-linear form of tire model and tire-ground interaction effects and proposes how this model can be effectively used for estimation of contingency risks and compensation of their influence upon the system. The aforementioned control structure ensures better system robustness to the system uncertainties of different type and better implementation capabilities of indoor mobile robots.

\section{Acknowledgement}

The results presented in the paper are part of the research project 'Research and Development of Ambientally Intelligent Service Robots of Anthropomorphic Characteristics', TR-35003 funded by Ministry of Science of Republic Serbia and partially funded by the Provincial Secretariat for Science and Technological Development of Autonomous Province of Vojvodina, Republic of Serbia, under the contract no. 114-451-2116/2011. Project is also supported by the SNSF SCOPES IP project 'CARE-robotics', ID: IZ74Z0_137361/1.

\section{References}

[1] Morin, P., Samson, C.: Motion Control of Wheeled Mobile Robots. In: Siciliano, B. and Khatib, O. (eds.) Handbook of Robotics, Springer, 2008, pp. $729-825$

[2] Minguez, J., Lamiraux, F., Laumond, J-P.: Motion Planning and Obstacle Avoidance. In: Siciliano, B. and Khatib, O. (eds) Handbook of Robotics Springer, 2008, pp. 826-850

[3] Lumelsky, V., Stepanov, A.: Path Planning Strategies for a Point Mobile Automation Moving Admist Unknown Obstacles of Arbitrary Shape. Algorithmica 2, 1987, pp. 403-430

[4] Khatib, O.: Real-Time Obstacle Avoidance for Manipulators and Mobile Robots. International Journal of Robotic Research 5, 1986, pp. 90-98

[5] Minguez, J., Montano, L.: Nearness Niagram (ND) Navigation: Collision Avoidance in Troublesome Scenarios. IEEE Transact. on Robot. Autom. 20(1), 2000, pp. 45-59

[6] Minguez, J., Osuna, J., Montano, L.: A Divide and Conquer Strategy to Achieve Reactive Collision Navigation Systems. IEEE Int. Conf. Robot. Autom., 2004, pp. 412-418 
[7] István Nagy, Behaviour Study of a Multi-Agent Mobile Robot System during Potential Field Building, Acta Polytechnica Hungarica, Vol. 6, No. 4, 2009, pp. 111-136

[8] Radu-Emil Precup, Stefan Preitl, Emil M. Petriu, József K. Tar, Marius L. Tomescu, Claudiu Pozna, Generic Two-Degree-of-Freedom Linear and Fuzzy Controllers for Integral Processes, Journal of Franklin Instituteengineering and Applied Mathematics, Vol. 346, No. 10, 2009, pp. 9801003

[9] Kovacs, S.; Vincze, D.; Gacsi, M.; Miklosi, A.; Korondi, P., Ethologically Inspired Robot Behavior Implementation, $4^{\text {th }}$ International Conference on Human System Interactions (HSI), 2011, pp. 64-69

[10] Károly Nagy, Szabolcs Divéki, Péter Odry, Matija Sokola, Vladimir Vujičić, A Stochastic Approach to Fuzzy Control, Acta Polytechnica Hungarica, Vol. 9, No. 6, 2012, pp. 29-48

[11] Aleksandar Rodić, Miomir Vukobratović: Dynamics, Integrated Control and Stability of Automated Road Vehicles. Ibidem Verlag, Stuttgart, 2002

[12] RobuLAB10 product sheet. Robosoft. http://www.robosoft.fr/img/data/ robuLAB10_web.pdf, Accessed May 2011

[13] Pacejka, H., Bakker, B.: The Magic Formula Tire Model. Vehicle System Dynamics, Vol. 21, 1993

[14] Aleksandar Rodic, Gyula Mester, "Virtual WRSN - Modeling and Simulation of Wireless Robot-Sensor Networked Systems". Proceedings of the $8^{\text {th }}$ IEEE International Symposium on Intelligent Systems and Informatics, SISY 2010, Subotica, Serbia, 2010, pp. 115-120

[15] Experimental testbed station for research and development of robot-sensor networked systems, Robotics Laboratory, Institute Mihailo Pupin, http://www.pupin.rs/RnDProfile/robots.html. Accesed January, 2013

[16] Gyula Mester, Intelligent Mobile Robot Motion Control in Unstructured Environments, Acta Polytechnica Hungarica, Journal of Applied Sciences, Vol. 7, Issue No. 4, Budapest, Hungary, 2010, pp. 153-165

[17] Aleksandar Rodić, Khalid Addi, Mirko Jezdimirović, Sensor-based Intelligent Navigation and Control of Autonomous Mobile Robots in Advanced Terrain Missions, Scientific Technical Review, Vol. 60, No. 2, 2010, pp. 7-15

\section{Appendix}

Kinematical and dynamic parameters of the industrial mobile robot platform RobuLab-10 [8] was assumed for modeling and simulation in this paper. Parameters are taken from the product sheet or estimated by use of the model. Control gains are synthesized in a manner described at the end of Appendix. Parameters used in the paper are presented in Table A1. Tire model coefficients $B_{j}, C_{j}, D_{j}, E_{j}, j=1,2,3$ whose values are given in Tab. A1, are determined from 
the corresponding empiric tire force graphs. Index $j=1$ is used for the longitudinal tire force model, $j=2$ for the lateral tire model and $j=3$ for the tire self-aligning torque model curves.

Control gains of the PD regulator to be used in (21) are determined by implementing of the Pole Placement Method.

Table A1

Kinematical, dynamic and control parameters used in simulation

\begin{tabular}{|c|c|c|}
\hline Parameter & Value & Unit \\
\hline$r_{t}$ & 0.05 & $\mathrm{~m}$ \\
\hline$l_{f}$ & 0.215 & $\mathrm{~m}$ \\
\hline$b$ & 0.360 & $\mathrm{~m}$ \\
\hline$l_{r}$ & 0.235 & $\mathrm{~m}$ \\
\hline$l_{x}$ & 0 & $\mathrm{~m}$ \\
\hline$m$ & 20 & $\mathrm{~kg}$ \\
\hline$I_{z}$ & 0.6042 & $k g m^{2}$ \\
\hline$g$ & 9.81 & $m / s^{2}$ \\
\hline$K_{x}$ & 1.36 & $N s^{2} / m^{2}$ \\
\hline$K_{y}$ & 1.50 & $N s^{2} / m^{2}$ \\
\hline$f_{r i}$ & 0.02 & \\
\hline$\mu_{s}$ & 0.75 & \\
\hline$B_{1}, B_{2}, B_{3}$ & $0.0985,0.1884,0.4966$ & \\
\hline$C_{1}, C_{2}, C_{3}$ & $1.65,1.30,2.40$ & \\
\hline$D_{1}, D_{2}, D_{3}$ & $38.84,38.84,12.00$ & $N$ \\
\hline$E_{1}, E_{2}, E_{3}$ & $(-7.4617,-2.8556,-0.1006) \times 10^{5} \times 10^{5}$ & \\
\hline$k_{p}$ & 355.3058 & $\mathrm{Nm} / \mathrm{rad}$ \\
\hline$k_{d}$ & 37.6991 & $\mathrm{Nms} / \mathrm{rad}$ \\
\hline
\end{tabular}

The frequency and the relative damping coefficient of the close-loop control system are chosen to be $f_{P D}=3.00(\mathrm{~Hz})$ and $\zeta_{P D}=1.00$. That ensures the poles of the control system are in the left plane. The following relations are used to determine the PD control gains (obtained values are given in Table A1):

$\varpi=2 \pi \cdot f_{P D}$

$k_{p}=\varpi^{2}, \quad k_{d}=2 \cdot \zeta_{P D} \cdot \varpi$

$K_{p}=\operatorname{diag}\left\{k_{p}\right\}, \quad K_{d}=\operatorname{diag}\left\{k_{d}\right\}$ 